\title{
NEW PLASTIC MICROINJECTION MOLDING TECHNIQUE FOR EXTREMELY TALL PLASTIC MICROSTRUCTURES USING REMOTE INFRARED RADIATION HEATING METHOD
}

\author{
Sanghyo Kim, Ramachandran Trichur, Gregory Beaucage* and Chong H. Ahn \\ Microsystems and BioMEMS Lab, *Dept. Material Science and Engineering, \\ University of Cincinnati, \\ Cincinnati, OH 45221-0030 \\ Byung H. Kim \\ Dept. Mechanical and Industrial Engineering, \\ University of Massachusetts, \\ Amherst, MA 01003-2210
}

\begin{abstract}
Microfabrication methods using polymers are becoming increasingly popular as low-cost and high volume production alternative to silicon or glass-based MEMS technologies. In this paper an innovative plastic microinjection molding technique using rapid thermal processing (RTP) for the fabrication of high aspect ratio plastic microstructures is reported. This technique focuses on decreasing the cycle time of injection molding of high aspect ratio microstructures and at the same time to achieve better fill and reproducibility of the micromold. In this work the RTP process has been implemented and characterized using Cyclic olefin copolymers (COC), Polycarbonate (PC) and Poly methyl methacrylate (PMMA) and high aspect ratio plastic structures (taller than $100 \mu \mathrm{m}$ ) such as plastic micro needles and fluidic micro channels have been realized.
\end{abstract}

\section{INTRODUCTION}

Micro electro-mechanical Systems (MEMS) technology has been applied to the field of biomedical engineering and related field in recent years and there is an increasing demand for miniaturization of various systemic components of biochip. There also have been intense activities to reduce fabrication cost and to increase volume of production to make biochemical detection systems commercially available, so polymer micro fabrication is becoming increasingly important [1].

Microinjection molding is a technique to fabricate precision microstructures using polymers at low cost and high volumes. However, fabrication of high aspect ratio (HAR) plastic microstructures has been considered as one of the most difficult tasks to accomplish without compromising in cycle time $[2, \mathbf{3}]$, because of the difficulty of injection fill-depth control at low molding block temperatures as shown in Figure 1. A complete fill of the high aspect ratio features in the mold cavity is almost impossible to achieve without sufficiently increasing the molding block temperature [4], to successfully achieve the flow of the polymer inside the mold cavity. This will lead to a huge increase in cycle time as the mold has to undergo heating and cooling cycle every time when a plastic part is made.

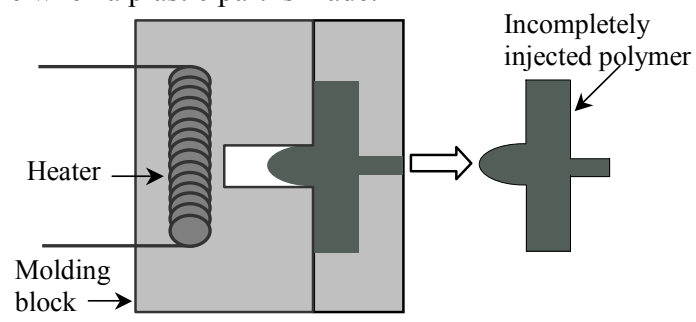

Figure 1. Illustration of an incompletely injected polymer part due to premature cooling inside the mold cavity.
In this paper an innovative technique of rapid thermal processing is discussed, where complete fill of the high aspect ratio features in the micro mold disk is achieved without compromising in cycle time, by increasing only the surface temperature of the mold insert by infrared radiation to the melting temperature of the plastic, rather than heating the bulk of the molding block.

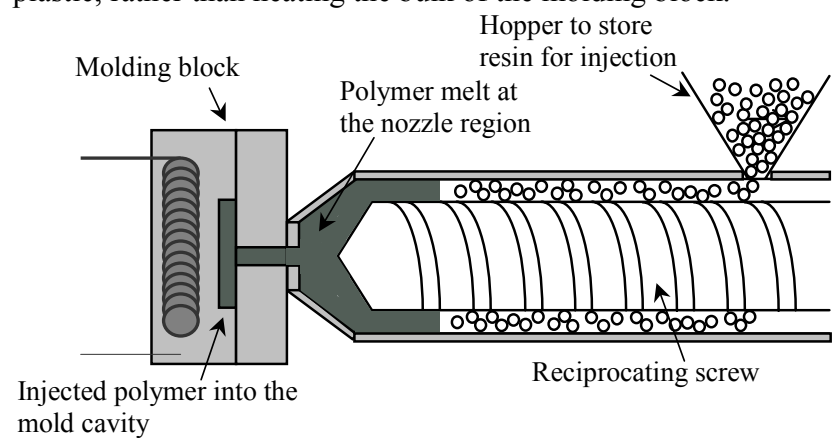

Figure 2. Schematic diagram of an injection molding machine with molding block.

\section{INJECTION MOLDING PROCESS}

The schematic of an injection molding machine is shown in Figure 2. With this machine the plastic is melted and then injected into the cavity of a closed mold, whose shape is transferred to plastic microstructures on cooling down. Inside the mold cavity the resin continues to flow and fill the mold cavity till the polymer cools down to a highly viscous melt, where the flow stops, and the part can be ejected. In order to ensure good flow properties during injection, thermoplastics with low or medium viscosity are preferred [5]. So the filling of the mold cavity and subsequently the micro patterns depend on viscosity of polymer melt, injection speed, molding block temperature and the nozzle temperature of the injection unit.

Thus, to achieve a good fill inside the high aspect ratio micro cavities in the mold cavity the molding materials are often processed at their maximum processing temperatures at the injection nozzle region. This ensures lower viscosity and better flow inside the mold cavity. Also the choice of a polymer with lower melt flow index (lower viscosity) and use of higher injection speeds help to achieve better filling inside the mold cavity.

The molding block is normally heated to an elevated temperature (below glass transition temperature of the injected polymer) to help uniform flow of polymer throughout the mold cavity and to prevent abrupt cooling of the polymer melt, which can drastically change its physical properties like mechanical strength, hardness, etc. But, in order to obtain better fill of the high aspect ratio structures inside the mold disk, the tool

Travel support has been generously provided by the Transducers Research

Foundation and by the DARPA MEMS and DARPA BioFlips programs. 
temperature can be raised even higher (above the glass transition temperature), but this drastically increases the cycle time to injection mold one single plastic part, which increases the manufacturing costs and also decreases throughput.

Thus, the reduction of cycle time is one of the most important issues that need to be addressed to achieve commercially viable and low cost micro structured plastic products. By the use of the rapid thermal processing technique, the cycle time is kept constant as of the conventional injection molding process, but a better fill of the high aspect ratio mold features can be achieved without heating the molding block to above glass transition temperatures.

\section{MOLD FABRICATION}

We have developed a new replaceable mold disk technique for the fabrication of the replaceable micro mold disk inserts for injection molding [6]. In this technique, the high aspect ratio microstructures are fabricated on a separate circular nickel disk about 3 inches in diameter and $1.5 \mathrm{~mm}$ in thickness. The disk is inserted in the molding block, as shown in Figure 3. This reduces many complexities of mold fabrication such as fabrication cost, handling, etc. The mold disk can be taken out from the molding block and be replaced with any other pattern without replacing the whole molding block. The replaceable mold disk technique also can go well with existing microfabrication techniques, which allows the replaceable disk molding technique to be a highly economically viable technique.
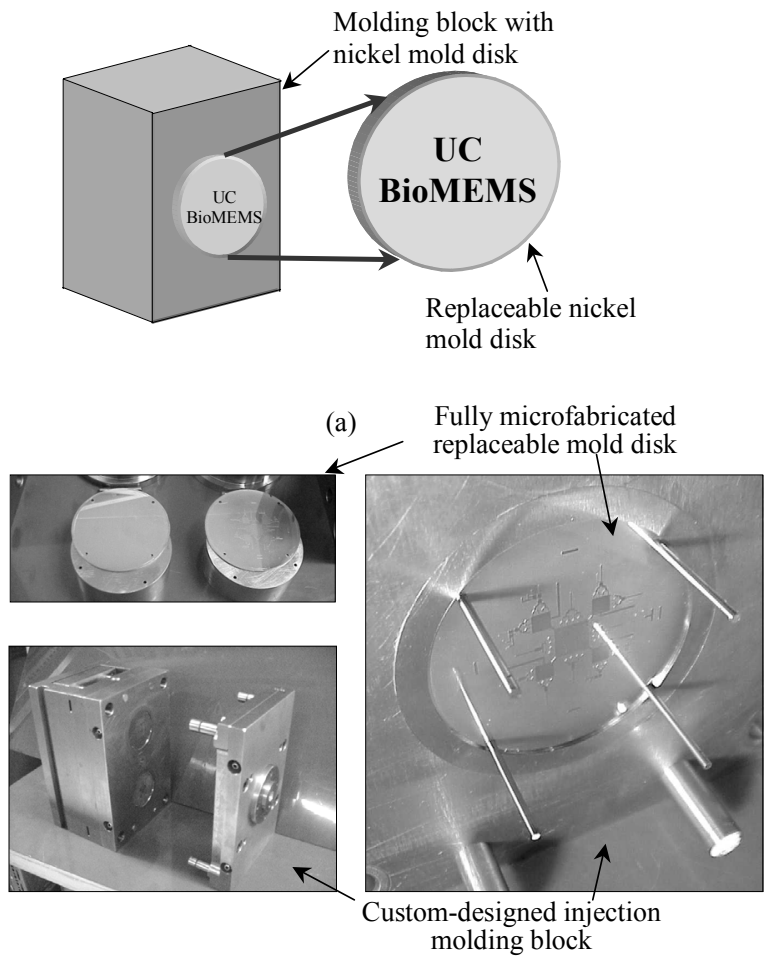

(b)

Figure 3.Injection molding block: (a) schematic of the molding block with the replaceable mold disk and (b) photographs of the custom designed molding block with the replaceable Ni mold disk and the fabricated microstructures in the Ni mold disk insert.

An essential step in the fabrication of the micro mold insert is surface preparation of nickel substrate, where the nickel substrate is lapped flat and parallel and polished using a polishing machine. The process summary is schematically described in Figure 4. (a)

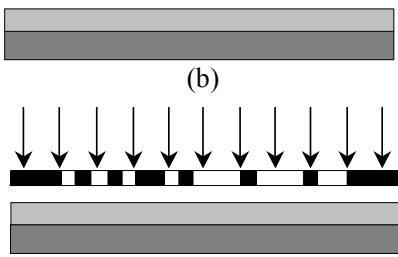

(c)

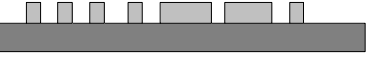

(d)

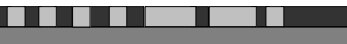

(e)

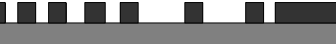

(f)
Figure 4. Schematic of microfabrication steps for the fabrication of Ni mold disk: (a) flat and parallel polished Ni substrate; (b) spin coated $S U 8$ on Ni substrate; (c) $U$-V exposure; (d) patterned SU 8 on nickel after developing; (e) electroplated nickel structures; and (f) final nickel mold disk with electroplated microstructures.

\section{RAPID THERMAL PROCESS FOR INJECTION MOLDING}

From the previous discussions, it is clear that to get a good fill of the polymer inside the mold disk cavity, the nozzle temperature should be near to the maximum processing temperature of the polymer and higher injection speeds and higher holding pressure should be used. But any attempt to increase the molding block temperature will drastically increase the cycle time of operation of the injection molding process.

In the rapid thermal process, the "surface" of a Ni mold disk is heated with IR radiation using a high power halogen lamp. The radiation from the IR source is focused to the surface of the Ni disk and the surface temperature of the replaceable Ni micromold disk reaches a temperature higher than the nozzle temperature in a few seconds. Then, the molding block is closed and the molten plastic is injected from the nozzle at high injection speeds. When the molten plastic enters the mold cavity, it experiences an isothermal environment. The melt temperature and the Ni mold disk surface temperature will be the same at the instant when the plastic is injected. So there will be no heat transfer taking place inside the cavity for a very small period of time and the surface heat of the $\mathrm{Ni}$ mold disk will help the plastic to be in a state of low viscosity. As a result of this, complete filling of the mold disk cavity can be achieved without any considerable increase in the cycle time of operation of the injection mold machine.
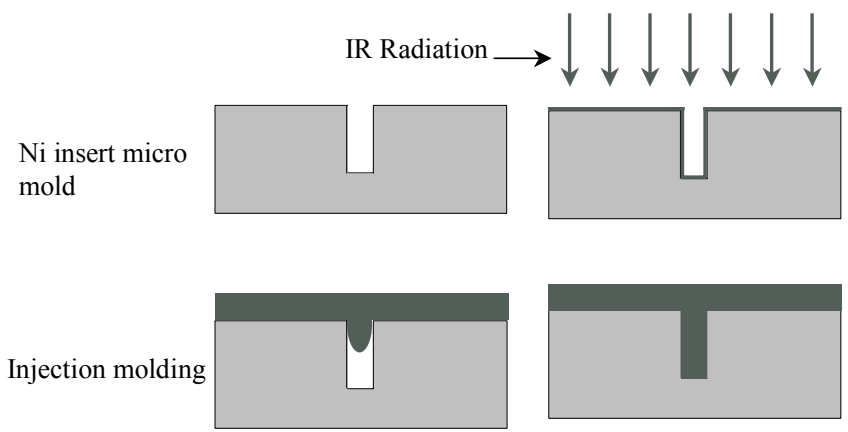

Final plastic part

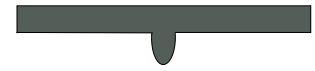

(a)

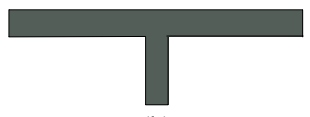

(b)
Figure 5. Comparison of the molding processes: (a) conventional molding and (b) rapid thermal processing. 


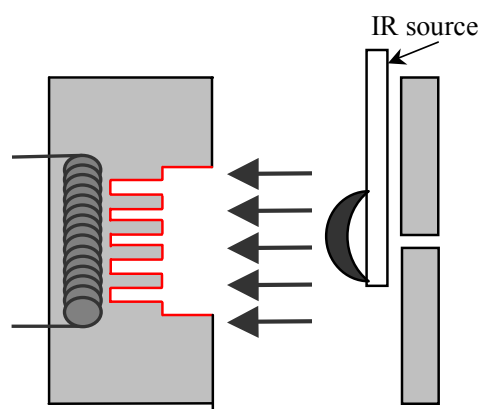

(a)

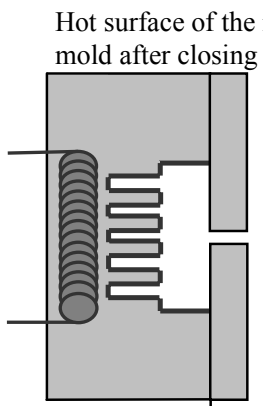

(b)

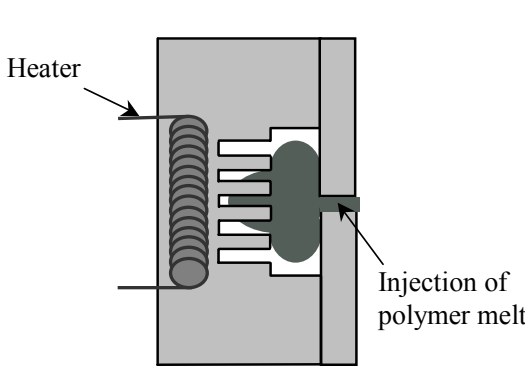

(c)
Completely filled and solidified polymer part

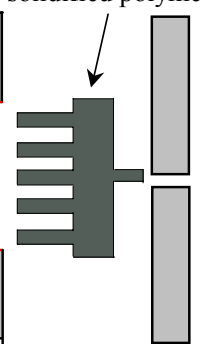

(d)

Figure 6. Schematic description of injection molding using rapid thermal process (a) surface heating of the mold disk using IR source; (b) closure of molding block; (c) filling phase of the injected polymer; and (d) complete filling, solidification and ejection of the final polymer part.

A comparison of the two processes and the differences of the fill depth in the final plastic microstructure are described in Figure 5. An illustration of the complete cycle of the rapid thermal process is summarized in Figure 6. The cycle time is almost similar to the conventional molding process, since only the surface of the micromold is heated, it reduces any need to heat and cool the whole molding block every time when a plastic part is made. Thus, the process using RTP with infrared radiation provides more economical and feasible source for large-scale production.

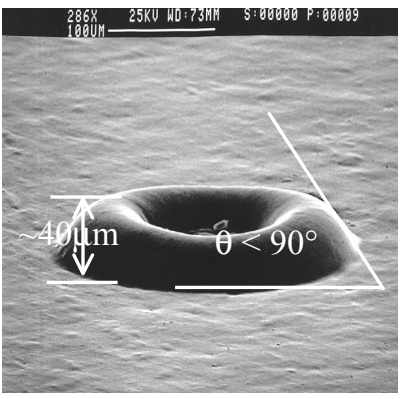

(a)

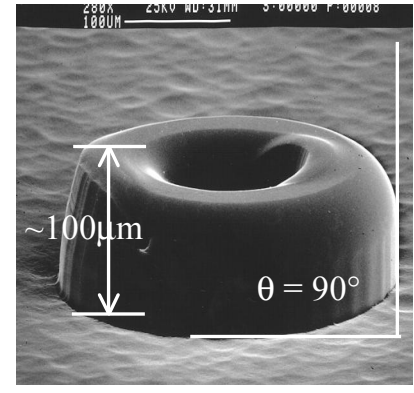

(b)
Figure 7. Comparison of the SEM pictures of microneedles: (a) without RTP process and (b) with RTP process.

SEM pictures of the actual microneedle structures are shown in Figure 7. The advantage of using the RTP process can be easily understood from the pictures. The shape of the microstructure without the RTP process is that of a partially filled micro cavity, where the plastic has solidified before complete filling of the mold cavity. The height of the structure is around $40 \mu \mathrm{m}$ and the sidewall profile has angle of around $45^{\circ}$. But the picture Figure 7 (b) of the microneedle fabricated using the RTP process shows complete filling of the mold and good replication of the microstructure in the mold cavity. The height of the plastic structure is around $100 \mu \mathrm{m}$, which is the depth of the micro cavity in the mold and the sidewall also has an angle of almost $90^{\circ}$, ensuring exact replication of the mold cavity. A picture of the microchannels fabricated using the rapid thermal process is shown in Figure 8, achieving complete replication of the mold.

A temperature profile of the surface of the Ni mold disk with respect to the exposure time to the IR radiation is shown in Figure 9. It can be seen from the graph that the surface temperature of the Ni mold disk can be raised from the molding block temperature to around $350{ }^{\circ} \mathrm{C}$ in less than 20 seconds. This ensures that the heat needed to maintain the flow of the polymer inside the micro mold disk cavity is attained quickly and the heat is dissipated quickly when the lamp is turned off, so the need for a cooling system is naturally averted.

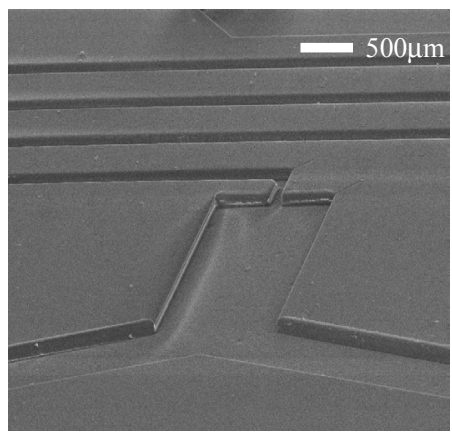

Figure 8. SEM picture of microchannels in plastic substrate fabricated by RTP process showing complete replication of the mold.

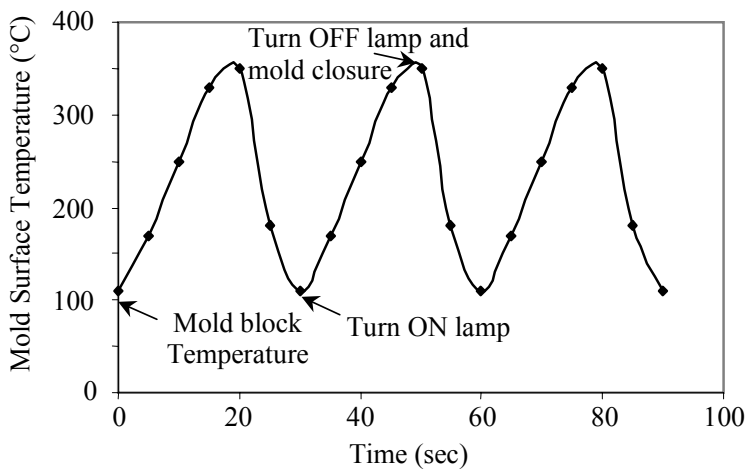

Figure 9. Surface temperature cycle of the Ni mold surface during $I R$ radiation on the mold surface.

\section{RESULTS AND DISCUSSION}

The rapid thermal process (RTP) was characterized for three different polymers such as Cyclic olefin copolymer (COC), Poly methyl methacrylate (PMMA) and Polycarbonate (PC). The RTP injection molding was performed on these three polymers with various injection speeds from $10 \mathrm{cc} / \mathrm{sec}$ to $70 \mathrm{cc} / \mathrm{sec}$ in steps of 20 $\mathrm{cc} / \mathrm{sec}$. The IR radiation exposure time was also varied from 10 secs to 2 minutes for all the polymers and for various injection speeds. The molding block temperature was fixed at two points, room temperature and maximum debinding temperature for each polymer, and the injection was performed at various speeds at these two temperatures. The RTP parameters were varied when the molding block was at debinding temperature of each polymer.

The results of experiment are plotted in Figure 10 for the polymers PMMA, PC and COC respectively. From the results, the 
maximum injection fill depth is achieved in most cases at around 40 seconds of IR radiation exposure time, which makes the cycle time of the injection mold machine around 90 seconds.

From the figures, complete fill of the mold has been never achieved at lower injection speeds, less than $30 \mathrm{cc} / \mathrm{sec}$, irrespective of the process and $100 \%$ fill has been achieved at speeds greater than $30 \mathrm{cc} / \mathrm{sec}$ and at exposure times around 40 seconds.

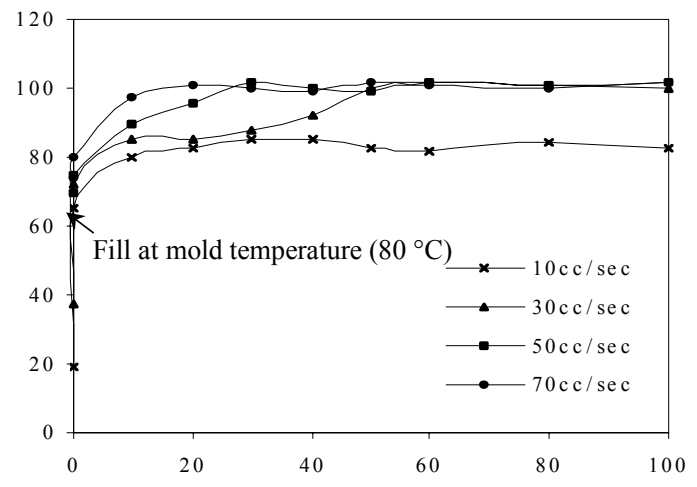

(a)

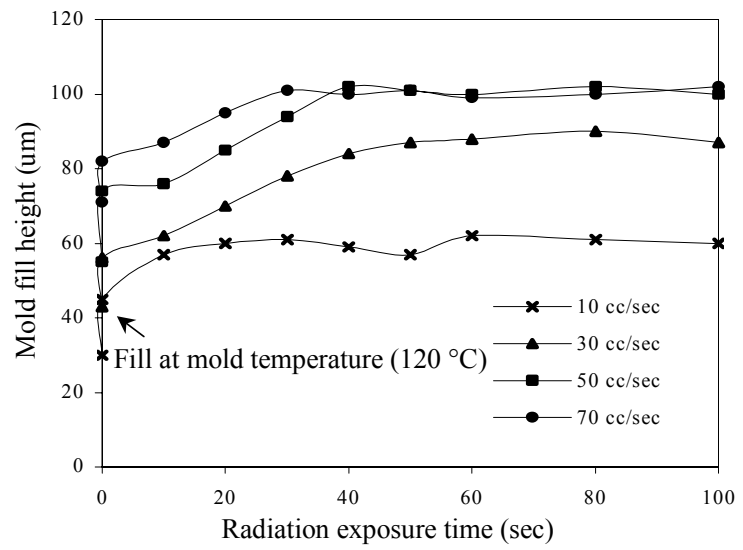

(b)

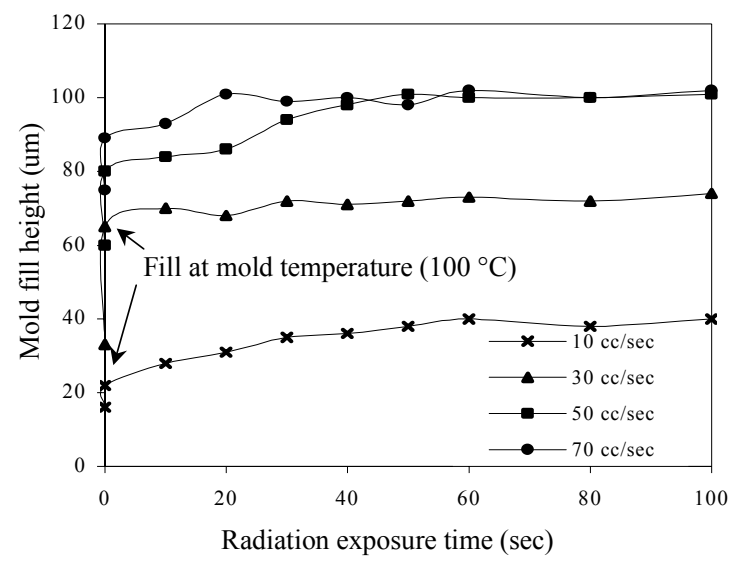

(c)

Figure 10. Measured injection fill depth at various IR exposure time, flow rates and molding block temperature: (a) Poly methyl methacrylate (PMMA); (b) Polycarbonate (PC); and (c) Cyclic olefin copolymer (COC).
The process can be greatly improved by including a thermal shield between the replaceable $\mathrm{Ni}$ mold disk and the molding block, since there is a huge temperature gradient between the disk surface and the molding block surface. Use of higher-powered IR source can greatly help to reduce the cycle time.

\section{CONCLUSION}

In this work, an innovative injection molding technique using rapid thermal process has been proposed, implemented and characterized using Cyclic olefin copolymer (COC), Polycarbonate (PC), and Poly methyl methacrylate (PMMA). The obtained results clearly revealed the advantages of the rapid thermal process. The cycle time for the full process was around 90 seconds. This process time clearly shows the advantage of the rapid thermal process over the conventional high aspect ratio injection molding, where the cycle time required was several minutes.

Thus the injection molding technique using the rapid thermal process is a favorable alternative to the conventional high aspect ratio injection molding and the cycle time can be greatly reduced, thereby reducing the injection molding costs and increasing the throughput of the process. The rapid thermal process allows the plastic micromachining using injection molding a commercially viable and economically feasible process for high volume production, which provides plastic biochips and biochemical detection systems to the end user at low cost.

\section{ACKNOWLEDGEMENT}

This research was fully supported by a DARPA grant under contract AF F30602-00-1-0569 from the BioFlips program, DoD, USA.

\section{REFERENCES}

[1] H. Becker and U. Heim, "Hot embossing as a method for the fabrication of polymer high aspect ratio structures", Sensors and Actuators (2000), 130-135.

[2] M. S. Despa, K. W. Kelly and J. R. Collier, "Injection molding of polymeric LIGA-HARMS", Microsystem Technologies (1999), 60-66.

[3] V. Piotter, T. Hanemann, R. Ruprecht, and J. Hau elt, "Injection molding and related techniques for fabrication of microstructures", Microsystem Technologies (1997), vol.3, 129-133.

[4] V. Piotter, R. Ruprecht, W. Bacher and J. H. Hausselt, "Injection molding of microstructures using filled and unfilled thermoplastics", proc. of the SPIE (1995), vol.2639, 146-57.

[5] V. Piotter, T. Gietzelt, T. Hanemann, R. Ruprecht, and J. Hausselt, "Materials for molding processes in microsystem technology", proc. Micro Materials (2000), 274-277.

[6] J. W. Choi, S. Kim, R. Trichur, H. J. Cho, A. Puntambekar, R. L. Cole, J. Simkins, S. Murugesan, K. Kim, J. B. Lee, G. Beaucage, J. H. Nevin, and C. H. Ahn, "A plastic micro injection molding technique using replaceable mold-disks for disposable microfluidic systems and biochips", proc. of $\mu T A S$ (2001), 411-412. 